\section{Federation University ResearchOnline}

\section{https://researchonline.federation.edu.au}

Copyright Notice

This is the author's preprint version of the following publication:

Barbagallo, Gala, S., Ragusa, M. A., \& Théra, M. (2020). On the Regularity of Weak Solutions of the Boussinesq Equations in Besov Spaces. Vietnam Journal of Mathematics, 49(3), 637-649.

The version displayed here may differ from the final published version.

The final publication is available at Springer via:

https://doi.org/10.1007/s10013-020-00420-4

Copyright ( ) Vietnam Academy of Science and Technology (VAST) and Springer Nature Singapore Pte Ltd. 


\title{
On the regularity of weak solutions of the Boussinesq equations in Besov spaces
}

\author{
A. Barbagallo · S. Gala · M. A. Ragusa · \\ Michel Théra
}

the date of receipt and acceptance should be inserted later

Abstract The main issue addressed in this paper concerns an extension of a result by Z. Zhang who proved, in the context of the homogeneous Besov space $\dot{B}_{\infty, \infty}^{-1}\left(\mathbb{R}^{3}\right)$, that, if the solution of the Boussinesq equation (1.1) below (starting with an initial data in $\left.H^{2}\right)$ is such that $(\nabla u, \nabla \theta) \in L^{2}\left(0, T ; \dot{B}_{\infty, \infty}^{-1}\left(\mathbb{R}^{3}\right)\right)$, then the solution remains smooth forever after $T$. In this contribution, we prove the same result for weak solutions just by assuming the condition on the velocity $u$ and not on the temperature $\theta$.

Part of the work was carried out while S. Gala was a long-term visitor at the University of Catania. The hospitality of Catania University is graciously acknowledged.

Research of M.A. Ragusa is partially supported by the Ministry of Education and Science of the Russian Federation (5-100 program of the Russian Ministry of Education).

Research of M. Théra is supported by the Australian Research Council (ARC) grant DP160100854 and benefited from the support of the FMJH Program PGMO and from the support of EDF.

First author

Department of Mathematics and Applications "R.Caccioppoli"

University of Naples 'Federico II", Naples

E-mail: annamaria.barbagallo@unina.it

Second author

Department of Sciences exactes, Ecole Normale Supérieure de Mostaganem

Box 227, Mostaganem 27000, Algeria

E-mail: sgala793@gmail.com

Third author

Dipartimento di Matematica e Informatica, Università di Catania and RUDN University, Moscow Russia Viale Andrea Doria, 695125 Catania - Italy

RUDN University, 6 Miklukho - Maklay St, Moscow, 117198, Russia

E-mail: maragusa@unict.it

Fourth author

XLIM UMR-CNRS 7252 Université de Limoges and Centre for Informatics and Applied Optimisation,

Federation University Australia

E-mail: michel.thera@unilim.fr 
Mathematics Subject Classification(2000): 35Q35, 35B65, 76D05.

Key words: Boussinesq equations, Besov space, weak solution, regularity criterion.

\section{Introduction}

We are interested in the regularity of weak solutions of the Cauchy problem related to the Boussinesq equations in $\mathbb{R}^{3}$ :

$$
\left\{\begin{array}{c}
\partial_{t} u+(u \cdot \nabla) u-\Delta u+\nabla \pi=\theta e_{3}, \\
\partial_{t} \theta+(u \cdot \nabla) \theta-\Delta \theta=0, \\
\nabla \cdot u=0, \\
u(x, 0)=u_{0}(x), \quad \theta(x, 0)=\theta_{0}(x),
\end{array}\right.
$$

where $x \in \mathbb{R}^{3}$ and $t \geq 0$. Here, $u: \mathbb{R}^{3} \times \mathbb{R}_{+} \rightarrow \mathbb{R}^{3}$ is the velocity field of the flow, $\pi=$ $\pi(x, t) \in \mathbb{R}$ is a scalar function representing the pressure, $\theta: \mathbb{R}^{3} \times \mathbb{R}_{+} \rightarrow \mathbb{R}^{3}$ represents the temperature of the fluid and $e_{3}=(0,0,1)^{T}$. Note that $u_{0}(x)$ and $\theta_{0}(x)$ are given initial velocity and initial temperature with $\nabla \cdot u_{0}=0$ in the sense of distributions.

Owing to the physical importance and the mathematical challenges, the study of (1.1) which describes the dynamics of a viscous incompressible fluid with heat exchanges, has a long history and has attracted many contributions from physicists and mathematicians [17]. Although Boussinesq equations consist in a simplification of the original 3-D incompressible flow, they share a similar vortex stretching effect. For this reason they retains most of the mathematical and physical difficulties of the 3-D incompressible flow, and therefore, these equations have been studied and applied to various fields. Examples include for instance geophysical applications, where they serve as a model, see, e.g. [19]. There are several other results on existence and blowup criteria in different kinds of spaces which have been obtained, (see [1,3, 7-9]).

The problem of the global-in-time well-posedness of (1.1) in a three-dimensional space is highly challenging, due to the fact that the system contains the incompressible 3D Navier-Stokes equations as a special case (obtained by setting $\theta=0$ ), for which the issue of global well-posedness has not been proved until now. However, the question of the regularity of weak solutions is an outstanding open problem in mathematical fluid mechanics and many interesting results have been obtained (see e.g. $[4,5,10-14,20,21,24-27])$. We are interested in the classical problem of finding sufficient conditions for weak solutions of (1.1) such that they become regular.

Realizing the dominant role played by the velocity field in the regularity issue, Ishimura and Morimoto [10] were able to derive criteria in terms of the velocity field $u$ alone. They showed that, if $u$ satisfies

$$
\nabla u \in L^{1}\left(0, T ; L^{\infty}\left(\mathbb{R}^{3}\right)\right),
$$

then the solution $(u, \theta)$ is regular on $[0, T]$. It is worthy to emphasize that there are no assumptions on the temperature $\theta$. This assumption (1.2) was weakened in [6] with the $L^{\infty}$-norm replaced by norms in Besov spaces $\dot{B}_{\infty, \infty}^{0}$. Quite recently, Z. Zhang [23] showed that $(u, \theta)$ is a strong solution if

$$
(\nabla u, \nabla \theta) \in L^{2}\left(0, T ; \dot{B}_{\infty, \infty}^{-1}\left(\mathbb{R}^{3}\right)\right),
$$


where $\dot{B}_{\infty, \infty}^{-1}$ denotes the homogenous Besov space. A logarithmically improvement of Zhang's result, controlled by its $H^{3}$-norm, was given by Ye [22].

The main purpose of this work is to establish an improvement of Zhang's regularity criterion (1.3). Now, the refined regularity criterion in terms of the gradient of the velocity $\nabla u$ can be stated as follows:

Theorem 1.1 (Main result) Assume that $\left(u_{0}, \theta_{0}\right) \in L^{2}\left(\mathbb{R}^{3}\right)$ with $\nabla \cdot u_{0}=0$. Let $(u, \theta)$ be a weak solution to the Boussinesq equations on some interval $(0, T)$ with $0<T \leq$ $\infty$. If

$$
\nabla u \in L^{2}\left(0, T ; \dot{B}_{\infty, \infty}^{-1}\left(\mathbb{R}^{3}\right)\right)
$$

then the weak solution $(u, \theta)$ is regular in $(0, T]$, that is $(u, \theta) \in C^{\infty}\left(\mathbb{R}^{3} \times(0, T]\right)$.

Remark 1.1 This result is expected because of the fact that the (refinement of) BealeKato-Majda type criterion is well known in the class $\dot{B}_{\infty, \infty}^{0}$ for the $3 D$ Boussinesq equations and one may replace the vorticity by $\nabla u$ since the Riesz transforms are continuous in $\dot{B}_{\infty, \infty}^{0}$. Then, the temperature plays a less dominant role than the velocity field does in the regularity theory of solutions to the Boussinesq equations. Furthermore, clearly Theorem 1.1 is an improvement of Zhang's regularity criterion (1.3).

By a weak solution, we mean that $(u, \theta, \pi)$ must satisfy (1.1) in the sense of distributions. In addition, we have the basic regularity for the weak solution

$$
(u, \theta) \in L^{\infty}\left(0, T ; L^{2}\left(\mathbb{R}^{3}\right)\right) \cap L^{2}\left(0, T ; H^{1}\left(\mathbb{R}^{3}\right)\right),
$$

for any $T>0$. If a weak solution $(u, \theta)$ satisfies

$$
(u, \theta) \in L^{\infty}\left(0, T ; H^{1}\left(\mathbb{R}^{3}\right)\right) \cap L^{2}\left(0, T ; H^{2}\left(\mathbb{R}^{3}\right)\right),
$$

then actually $(u, \theta)$ is a strong (classical) solution. It is worth to note that for strong solutions, we can gain more regularity properties.

Throughout this paper, $C$ denotes a generic positive constant which may vary from one line to another.

\section{Preliminaries}

In this section we introduce the function spaces that will be used to state and prove the main result, and we collect and/or derive a number of auxiliary estimates that will be needed throughout the proof. Before introducing the homogeneous Besov and Triebel-Lizorkin spaces, we have to fix some notations. By $\mathscr{S}$ we denote the class of rapidly decreasing functions. The dual space of $\mathscr{S}$, i.e., the space of tempered distributions on $\mathbb{R}^{3}$ is denoted by $\mathscr{S}^{\prime}$. For $u \in \mathscr{S}\left(\mathbb{R}^{3}\right)$, the Fourier transform of $u$ is defined by

$$
\mathscr{F} u(\omega)=\widehat{u}(\omega)=\int_{\mathbb{R}^{3}} u(x) e^{-i x \cdot \omega} d x, \quad \omega \in \mathbb{R}^{3}
$$


The homogeneous Littlewood-Paley decomposition relies upon a dyadic partition of unity. We can use for instance any $\varphi \in \mathscr{S}\left(\mathbb{R}^{3}\right)$, supported in $\mathscr{C} \triangleq\left\{\omega \in \mathbb{R}^{3}: \frac{3}{4} \leq|\omega| \leq \frac{8}{3}\right\}$ such that

$$
\sum_{l \in \mathbb{Z}} \varphi\left(2^{-l} \omega\right)=1 \text { if } \omega \neq 0 .
$$

Denoting $h=\mathscr{F}^{-1} \varphi$, we then define dyadic blocks in this way:

$$
\Delta_{l} u \triangleq \varphi\left(2^{-l} D\right) u=2^{3 l} \int_{\mathbb{R}^{3}} h\left(2^{l} y\right) u(x-y) d y, \quad \text { for each } l \in \mathbb{Z},
$$

and

$$
S_{l} u \triangleq \sum_{k \leq l-1} \Delta_{k} u
$$

The formal decomposition

$$
u=\sum_{l \in \mathbb{Z}} \Delta_{l} u
$$

is called the homogeneous Littlewood-Paley decomposition.

Remark 2.1 The above dyadic decomposition has nice properties of quasi-orthogonality: with our choice of $\varphi$, we have,

$$
\Delta_{k} \Delta_{l} u \equiv 0 \text { if }|k-l| \geq 2 \text { and } \Delta_{k}\left(S_{k-1} u \Delta_{l} u\right) \equiv 0 \text { if }|k-l| \geq 5 .
$$

With the introduction of $\Delta_{l}$, let us recall the definition of homogeneous Besov and Triebel-Lizorkin spaces (see [23] for more details).

Definition 2.1 The homogeneous Besov space $\dot{B}_{p, q}^{s}\left(\mathbb{R}^{3}\right)$ is defined by

$$
\dot{B}_{p, q}^{s}\left(\mathbb{R}^{3}\right)=\left\{u \in \mathscr{S}^{\prime}\left(\mathbb{R}^{3}\right) / \mathscr{P}\left(\mathbb{R}^{3}\right):\|u\|_{\dot{B}_{p, q}^{s}}<\infty\right\},
$$

for $s \in \mathbb{R}$ and $1 \leq p, q \leq \infty$, where

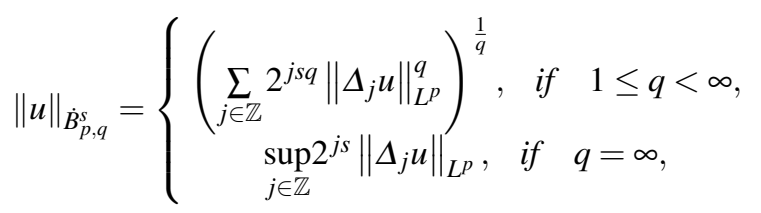

and $\mathscr{P}\left(\mathbb{R}^{3}\right)$ is the set of all scalar polynomials defined on $\mathbb{R}^{3}$. Similarly, the homogeneous Triebel-Lizorkin spaces $\vec{F}_{p, q}\left(\mathbb{R}^{3}\right)$ is a quasi-normed space equipped with the family of semi-norms $\|\cdot\|_{F_{p, q}}$ which are defined by

$$
\|u\|_{F_{p, q}}=\left\{\begin{array}{c}
\left\|\left(\sum_{j \in \mathbb{Z}} 2^{j s q}\left|\Delta_{j} u\right|^{q}\right)^{\frac{1}{q}}\right\|_{L^{p}}, \quad \text { if } 1 \leq q<\infty, \\
\left\|\sup _{j \in \mathbb{Z}} 2^{j s}\left|\Delta_{j} u\right|\right\|_{L^{p}}, \quad \text { if } \quad q=\infty .
\end{array}\right.
$$


Notice that there exists a universal constant $C$ such that

$$
C^{-1}\|u\|_{\dot{B}_{p, q}^{s}} \leq\|\nabla u\|_{\dot{B}_{p, q}^{s-1}} \leq C\|u\|_{\dot{B}_{p, q}^{s}} .
$$

In particular,

$$
u \in \dot{B}_{\infty, \infty}^{0}\left(\mathbb{R}^{3}\right) \Longleftrightarrow \nabla u \in \dot{B}_{\infty, \infty}^{-1}\left(\mathbb{R}^{3}\right) .
$$

From this observation we derive the following corollary to Theorem 1.1.

Corollary 2.1 Suppose that $(u, \theta)$ is a weak solution of the Boussinesq equations on $(0, T)$. If

$$
u \in L^{2}\left(0, T ; \dot{B}_{\infty, \infty}^{0}\left(\mathbb{R}^{3}\right)\right),
$$

then the weak solution $(u, \theta)$ is regular in $(0, T]$.

Next, we introduce the following Bernstein lemma due to [6].

Lemma 2.1 (Bernstein) For all $k \in \mathbb{N}, j \in \mathbb{Z}$, and $1 \leq p \leq q \leq \infty$, we have for all $f \in \mathscr{S}\left(\mathbb{R}^{3}\right)$ :

(i)

$$
\sup _{|\alpha|=k}\left\|\nabla^{\alpha} \Delta_{j} f\right\|_{L^{q}} \leq C_{1} 2^{j k+3 j\left(\frac{1}{p}-\frac{1}{q}\right)}\left\|\Delta_{j} f\right\|_{L^{p}}
$$

(ii)

$$
\left\|\Delta_{j} f\right\|_{L^{p}} \leq C_{2} 2^{-j k} \sup _{|\alpha|=k}\left\|\nabla^{\alpha} \Delta_{j} f\right\|_{L^{p}}
$$

where $C_{1}, C_{2}$ are positive constants independent of $f$ and $j$.

The proof of the main result needs a logarithmic Sobolev inequality in terms of Besov space. It will play an important role in the proof of Theorem 1.1. The following is a well-known embedding result, (cf. [23], pp. 244):

$$
L^{\infty}\left(\mathbb{R}^{3}\right) \hookrightarrow B M O\left(\mathbb{R}^{3}\right)=\dot{F}_{\infty, 2}^{0} \hookrightarrow \dot{B}_{\infty, \infty}^{0}\left(\mathbb{R}^{3}\right),
$$

where $B M O\left(\mathbb{R}^{3}\right)$ stands for the Bounded Mean Oscillations space [23].

We state and prove the following lemma.

Lemma 2.2 Suppose that $\nabla f \in \dot{B}_{\infty, \infty}^{-1}\left(\mathbb{R}^{3}\right)$ and $f \in H^{s}\left(\mathbb{R}^{3}\right)$ for all $s>\frac{3}{2}$. Then, there exists a constant $C>0$ such that

$$
\|f\|_{L^{\infty}} \leq C\left[1+\|\nabla f\|_{\dot{B}_{\infty, \infty}^{-1}}\left(\ln ^{+}\|f\|_{H^{s}}\right)^{\frac{1}{2}}\right],
$$

holds, where $H^{s}$ denotes the standard Sobolev space and

$$
\ln ^{+} x=\left\{\begin{array}{c}
\ln x, \text { if } x>e \\
1, \text { if } 0<x \leq e .
\end{array}\right.
$$


Proof: The proof is an easy modification of the one in [15]. Owing the LittlewoodPaley decomposition, we can rewrite

$$
f=\sum_{j \in \mathbb{Z}} \Delta_{j} f=\sum_{j=-\infty}^{-N-1} \Delta_{j} f+\sum_{j=-N}^{N} \Delta_{j} f+\sum_{j=N+1}^{+\infty} \Delta_{j} f,
$$

where $N$ is a positive integer to be determined later. Bernstein's lemma and Young's inequality give rise to

$$
\begin{aligned}
\|f\|_{L^{\infty}} & \leq \sum_{j=-\infty}^{-N-1}\left\|\Delta_{j} f\right\|_{L^{\infty}}+\sum_{j=-N}^{N}\left\|\Delta_{j} f\right\|_{L^{\infty}}+\sum_{j=N+1}^{+\infty}\left\|\Delta_{j} f\right\|_{L^{\infty}} \\
& \leq C \sum_{j<-N} 2^{\frac{3}{2} j}\left\|\Delta_{j} f\right\|_{L^{2}}+C N\|f\|_{\dot{B}_{\infty, \infty}^{0}}+C \sum_{j>N} 2^{\left(-s+\frac{3}{2}\right) j}\left\|\Delta_{j} f\right\|_{L^{2}} 2^{j s} \\
& \leq C\left(2^{-\frac{3}{2} N}\|f\|_{L^{2}}+N\|\nabla f\|_{\dot{B}_{\infty, \infty}^{-1}}+\sum_{j>N} 2^{\left(-s+\frac{3}{2}\right) j}\|f\|_{\dot{B}_{2, \infty}^{s}}\right) \\
& \leq C\left(2^{-\frac{3}{2} N}\|f\|_{L^{2}}+N\|\nabla f\|_{\dot{B}_{\infty, \infty}^{-1}}+2^{\left(-s+\frac{3}{2}\right) N}\|f\|_{H^{s}}\right)
\end{aligned}
$$

where we have used the fact that $s>\frac{3}{2}$ and the Besov embedding $H^{s} \hookrightarrow \dot{B}_{2, \infty}^{s}$.

Setting $\alpha=\min \left(s-\frac{3}{2}, \frac{3}{2}\right)$, we derive

$$
\|f\|_{L^{\infty}} \leq C\left(2^{-\alpha N}\|f\|_{H^{s}}+N\|\nabla f\|_{\dot{B}_{\infty, \infty}^{-1}}\right) .
$$

Now choose $N$ such that $2^{-\alpha N}\|f\|_{H^{s}} \leq 1$. Thus we get

$N \geq \frac{\log \|f\|_{H^{s}}}{\alpha \log 2}$.

Next, the following lemma is needed.

Lemma 2.3 Let $g, h \in H^{1}\left(\mathbb{R}^{3}\right)$ and $f \in B M O\left(\mathbb{R}^{3}\right)$. Then we have

$$
\int_{\mathbb{R}^{3}} f \cdot \nabla(g h) d x \leq C\|f\|_{B M O}\left(\|\nabla g\|_{L^{2}}\|h\|_{L^{2}}+\|g\|_{L^{2}}\|\nabla h\|_{L^{2}}\right) .
$$

Proof: The proof of the above lemma requires some paradifferential calculus. We have to recall here that paradifferential calculus enables to define a generalized product between distributions. It is continuous in many functional spaces where the usual product does not make sense (see the pioneering work of J.-M. Bony in [2]). The paraproduct between $f$ and $g$ is defined by

$$
T_{f} g \triangleq \sum_{j \in \mathbb{Z}} S_{j-1} f \Delta_{j} g
$$

We thus have the following formal decomposition (modulo a polynomial):

$$
f g=T_{f} g+T_{g} f+R(f, g)
$$

with

$$
R(f, g)=\sum_{|j-k| \leq 1} \Delta_{j} f \Delta_{k} g
$$


Coming back to the proof of Lemma 2.3 , we split $\int_{\mathbb{R}^{3}} f \cdot \nabla(g h) d x$ into

$$
\begin{aligned}
\int_{\mathbb{R}^{3}} f \cdot \nabla(g h) d x & =\int_{\mathbb{R}^{3}} f \cdot \nabla\left(T_{g} h\right) d x+\int_{\mathbb{R}^{3}} f \cdot \nabla\left(g T_{h}\right) d x+\int_{\mathbb{R}^{3}} f \cdot \nabla R(g, h) d x \\
& =I_{1}+I_{2}+I_{3} .
\end{aligned}
$$

Since we know that $B M O=\dot{F}_{\infty, 2}$ (see pp. 243-244 of [23]), the duality between $\dot{F}_{\infty, 2}$ and $\dot{F}_{1,2}$ guarantees that

$$
\begin{aligned}
I_{1} & =\int_{\mathbb{R}^{3}} f \cdot\left(T_{\nabla g} h\right) d x+\int_{\mathbb{R}^{3}} f \cdot\left(T_{g} \nabla h\right) d x \\
& \leq\|f\|_{B M O}\left(\left\|T_{\nabla g} h\right\|_{F_{1,2}}+\left\|T_{g} \nabla h\right\|_{F_{1,2}}\right) \\
& =\|f\|_{B M O}\left(I_{11}+I_{12}\right) .
\end{aligned}
$$

In view of the boundedness of the Hardy-Littlewood maximal operator $\mathscr{M}$ in $L^{p}$ spaces $(1<p<\infty)$ (c.f. Stein [ [22], Chap II, Theorem 1]), we can estimate the term $I_{11}$ as follows :

$$
\begin{aligned}
I_{11} & \approx\left\|\left(\sum_{j \in \mathbb{Z}}\left|S_{j-1}(\nabla g)\right|^{2}\left|\Delta_{j} h\right|^{2}\right)^{\frac{1}{2}}\right\|_{L^{1}} \leq C\left\|\mathscr{M}(\nabla g)\left(\sum_{j \in \mathbb{Z}}\left|\Delta_{j} h\right|^{2}\right)^{\frac{1}{2}}\right\|_{L^{1}} \\
& \leq C\|\mathscr{M}(\nabla g)\|_{L^{2}}\left\|\left(\sum_{j \in \mathbb{Z}}\left|\Delta_{j} h\right|^{2}\right)^{\frac{1}{2}}\right\|_{L^{2}} \leq C\|\nabla g\|_{L^{2}}\|h\|_{L^{2}} .
\end{aligned}
$$

Repeating the same arguments, we also have for $I_{12}$

$$
\begin{aligned}
I_{12} & \approx\left\|\left(\sum_{j \in \mathbb{Z}}\left|S_{j-1}(g)\right|^{2}\left|\Delta_{j}(\nabla h)\right|^{2}\right)^{\frac{1}{2}}\right\|_{L^{1}} \leq C\left\|\mathscr{M}(g)\left(\sum_{j \in \mathbb{Z}}\left|\Delta_{j}(\nabla h)\right|^{2}\right)^{\frac{1}{2}}\right\|_{L^{1}} \\
& \leq C\|g\|_{L^{2}}\left\|\left(\sum_{j \in \mathbb{Z}}\left|\Delta_{j}(\nabla h)\right|^{2}\right)^{\frac{1}{2}}\right\|_{L^{2}} \leq C\|g\|_{L^{2}}\|\nabla h\|_{L^{2}} .
\end{aligned}
$$

Collecting these estimates, we obtain

$$
I_{1} \leq C\|f\|_{B M O}\left(\|\nabla g\|_{L^{2}}\|h\|_{L^{2}}+\|g\|_{L^{2}}\|\nabla h\|_{L^{2}}\right) .
$$

As a result, estimating $I_{2}$ following the same arguments, we obtain

$$
I_{2} \leq C\|f\|_{B M O}\left(\|\nabla g\|_{L^{2}}\|h\|_{L^{2}}+\|g\|_{L^{2}}\|\nabla h\|_{L^{2}}\right) .
$$


For the third term $I_{3}$, using the embedding relation $\dot{B}_{1,1}^{0} \subset \dot{F}_{1,2}^{0}$ and in view of Bernstein's lemma, we can deduce that

$$
\begin{aligned}
I_{3} & \leq\|f\|_{B M O}\|\nabla R(g, h)\|_{F_{1,2}} \leq C\|f\|_{B M O}\|R(g, h)\|_{\dot{B}_{1,1}^{1}} \\
& \leq C\|f\|_{B M O} \sum_{j \in \mathbb{Z}} 2^{j}\left\|\Delta_{j} g \cdot \widetilde{\Delta}_{j} h\right\|_{L^{1}} \\
& \leq C\|f\|_{B M O} \sum_{j \in \mathbb{Z}} 2^{j}\left\|\Delta_{j} g\right\|_{L^{2}}\left\|\widetilde{\Delta}_{j} h\right\|_{L^{2}} \\
& \leq C\|f\|_{B M O}\|\nabla g\|_{L^{2}}\|h\|_{L^{2}} .
\end{aligned}
$$

so that the proof of Lemma 2.3 is achieved.

We often use the following well-known lemma.

Lemma 2.4 (Gagliardo-Nirenberg) Let $1 \leq q, r<\infty$ and $m \leq k$. Suppose that $\theta$ and $j$ satisfy $m \leq j \leq k, 0 \leq \theta \leq 1$ and define $p \in[1,+\infty]$ by

$$
\frac{1}{p}=\frac{j}{3}+\theta\left(\frac{1}{r}-\frac{m}{3}\right)+(1-\theta)\left(\frac{1}{q}-\frac{k}{3}\right) .
$$

Then, the inequality

$$
\left\|\nabla^{j} f\right\|_{L^{p}} \leq C\left\|\nabla^{m} f\right\|_{L^{q}}^{1-\theta}\left\|\nabla^{k} f\right\|_{L^{r}}^{\theta} \quad \text { for } f \in W^{m, q}\left(\mathbb{R}^{3}\right) \cap W^{k, r}\left(\mathbb{R}^{3}\right),
$$

holds with some constant $C>0$.

\section{Proof of Theorem 1.1}

Now we are ready to prove our main result of this section.

Proof: First, note that a weak solution $(u, \theta)$ to (1.1) has at least one global weak solution

$$
(u, \theta) \in L^{\infty}\left(0, T ; L^{2}\left(\mathbb{R}^{3}\right)\right) \cap L^{2}\left(0, T ; H^{1}\left(\mathbb{R}^{3}\right)\right),
$$

which satisfies the following energy inequality

$$
\begin{aligned}
& \frac{1}{2}\left(\|u(\cdot, t)\|_{L^{2}}^{2}+\|\theta(\cdot, t)\|_{L^{2}}^{2}\right)+\int_{0}^{t}\left(\|\nabla u(\cdot, \tau)\|_{L^{2}}^{2}+\|\nabla \theta(\cdot, \tau)\|_{L^{2}}^{2}\right) d \tau \\
\leq & \frac{1}{2}\left(\left\|u_{0}\right\|_{L^{2}}^{2}+\left\|\theta_{0}\right\|_{L^{2}}^{2}\right),
\end{aligned}
$$

for almost every $t \geq 0$.

In order to prove that $(u, \theta) \in C^{\infty}\left(\mathbb{R}^{3} \times(0, T]\right)$, as it is well known, it suffices to show that the weak solution $(u, \theta)$ is also a strong solution on $(0, T]$, which means that:

$$
(u, \theta) \in L^{\infty}\left(0, T ; H^{1}\left(\mathbb{R}^{3}\right)\right) \cap L^{2}\left(0, T ; H^{2}\left(\mathbb{R}^{3}\right)\right) .
$$


Owing to (1.4), we know that for any small constant $\varepsilon>0$, there exists $T_{0}=T_{0}(\varepsilon)<T$ such that

$$
\int_{T_{0}}^{T}\|\nabla u(\cdot, \tau)\|_{\dot{B}_{\infty, \infty}^{-1}}^{2} d \tau \leq \varepsilon
$$

To do so, we shall work on the local strong solution with the initial datum $\left(u_{0}, \theta_{0}\right)$ on its maximal existence time interval $\left(0, T_{0}\right)$. Then, we have only to show that

$\sup _{0 \leq t<T_{0}}\left(\|\nabla u(\cdot, t)\|_{L^{2}}^{2}+\|\nabla \theta(\cdot, t)\|_{L^{2}}^{2}\right)+\int_{0}^{T_{0}}\left(\|\nabla u(\cdot, \tau)\|_{L^{2}}^{2}+\|\nabla \theta(\cdot, \tau)\|_{L^{2}}^{2}\right) d \tau \leq C<\infty$,

here and in what follows $C$ denotes various positive constants which are independent from $T_{0}$.

Take the operator $\nabla$ in equations $(1.1)_{1}$ and $(1.1)_{2}$, respectively, and the scalar product of them $\nabla u$ and $\nabla \theta$, respectively and add them together, to obtain

$$
\begin{aligned}
& \frac{1}{2} \frac{d}{d t}\left(\|\nabla u\|_{L^{2}}^{2}+\|\nabla \theta\|_{L^{2}}^{2}\right)+\|\Delta u\|_{L^{2}}^{2}+\|\Delta \theta\|_{L^{2}}^{2} \\
= & -\int_{\mathbb{R}^{3}} \theta e_{3} \cdot \Delta u d x-\sum_{i=1}^{3} \int_{\mathbb{R}^{3}}\left(\partial_{i} u \cdot \nabla\right) u \partial_{i} u d x-\sum_{i=1}^{3} \int_{\mathbb{R}^{3}}\left(\partial_{i} u \cdot \nabla\right) \theta \partial_{i} \theta d x \\
: & =I_{1}+I_{2}+I_{3} .
\end{aligned}
$$

In the following, we estimate each term at the right-hand side of (3.12) separately below.

To bound $I_{1}$, we integrate by parts and apply Hölder's inequality to obtain

$$
\left|I_{1}\right| \leq C\|\nabla u\|_{L^{2}}\|\nabla \theta\|_{L^{2}} \leq C\left(\|\nabla u\|_{L^{2}}^{2}+\|\nabla \theta\|_{L^{2}}^{2}\right) .
$$

In order to deal with the terms $I_{2}$ and $I_{3}$, we need the following elegant MachiharaOzawa inequality [16] (see also Meyer [18])

$$
\|\nabla u\|_{L^{4}}^{2} \leq C\|u\|_{\dot{B}_{\infty, \infty}^{0}}\|\Delta u\|_{L^{2}} .
$$

We now bound $I_{2}$. By (3.13) and Young's inequality

$$
\begin{aligned}
\left|I_{2}\right| & \leq C\|\nabla u\|_{L^{2}}\|\nabla u\|_{L^{4}}^{2} \\
& \leq C\|\nabla u\|_{L^{2}}\|u\|_{\dot{B}_{\infty, \infty}^{0}}\|\Delta u\|_{L^{2}} \\
& \leq C\|\nabla u\|_{L^{2}}\|u\|_{B M O}\|\Delta u\|_{L^{2}} \\
& \leq \frac{1}{2}\|\Delta u\|_{L^{2}}^{2}+C\|\nabla u\|_{L^{2}}^{2}\|u\|_{B M O}^{2} .
\end{aligned}
$$

By integration by parts, we can rewrite and estimate $I_{3}$ as follows

$$
\begin{aligned}
\left|I_{3}\right| & =\left|\sum_{i=1}^{3} \int_{\mathbb{R}^{3}}\left(\partial_{i} u \cdot \nabla\right) \theta \cdot \partial_{i} \theta d x\right|=\left|\sum_{i, j, k=1}^{3} \int_{\mathbb{R}^{3}} \partial_{i}\left(\partial_{i} \theta_{k} \partial_{k} \theta_{j}\right) u_{j} d x\right| \\
& \leq C\|u\|_{B M O}\|\nabla \theta\|_{L^{2}}\|\Delta \theta\|_{L^{2}} \\
& \leq \frac{1}{6}\|\Delta \theta\|_{L^{2}}^{2}+C\|u\|_{B M O}^{2}\|\nabla \theta\|_{L^{2}}^{2} .
\end{aligned}
$$


Combining the estimates for $I_{1}, I_{2}$ and $I_{3}$, we find

$$
\begin{aligned}
& \frac{d}{d t}\left(\|\nabla u\|_{L^{2}}^{2}+\|\nabla \theta\|_{L^{2}}^{2}\right)+\|\Delta u\|_{L^{2}}^{2}+\|\Delta \theta\|_{L^{2}}^{2} \\
\leq & C\left(1+\|u\|_{B M O}^{2}\right)\left(\|\nabla u\|_{L^{2}}^{2}+\|\nabla \theta\|_{L^{2}}^{2}\right) .
\end{aligned}
$$

Using the Gronwall inequality on the time interval $\left[T_{0}, t\right]$, one has the following inequality

$$
\begin{aligned}
& \|\nabla u(\cdot, t)\|_{L^{2}}^{2}+\|\nabla \theta(\cdot, t)\|_{L^{2}}^{2}+\int_{T_{0}}^{t}\left(\|\Delta u(\cdot, \tau)\|_{L^{2}}^{2}+\|\Delta \theta(\cdot, \tau)\|_{L^{2}}^{2}\right) d \tau \\
\leq & \left(\left\|\nabla u\left(\cdot, T_{0}\right)\right\|_{L^{2}}^{2}+\left\|\nabla \theta\left(\cdot, T_{0}\right)\right\|_{L^{2}}^{2}\right) \exp \left(C \int_{T_{0}}^{t}\|u(\cdot, \tau)\|_{B M O}^{2} d \tau\right) .
\end{aligned}
$$

Let us denote for any $t \in\left[T_{0}, T\right)$,

$$
F(t) \triangleq \max _{T_{0} \leq \tau \leq t}\left(\|u(\cdot, \tau)\|_{H^{2}}^{2}+\|\theta(\cdot, \tau)\|_{H^{2}}^{2}\right)
$$

It should be noted that the function $F(t)$ is nondecreasing. Using (2.6), we obtain

$$
\begin{aligned}
& \|\nabla u(\cdot, t)\|_{L^{2}}^{2}+\|\nabla \theta(\cdot, t)\|_{L^{2}}^{2}+\int_{T_{0}}^{t}\left(\|\Delta u(\cdot, \tau)\|_{L^{2}}^{2}+\|\Delta \theta(\cdot, \tau)\|_{L^{2}}^{2}\right) d \tau \\
\leq & C\left(T_{0}\right) \exp \left(C \int_{T_{0}}^{t}\left(1+\|u(\cdot, \tau)\|_{\dot{B}_{\infty, \infty}^{0}}^{2} \log \left(\|u(\cdot, \tau)\|_{H^{2}}+\|\theta(\cdot, \tau)\|_{H^{2}}\right)\right) d \tau\right) \\
\leq & C\left(T_{0}\right) \exp \left(C \int_{T_{0}}^{t}\|\nabla u(\cdot, \tau)\|_{\dot{B}_{\infty, \infty}^{-1}}^{2} \log \left(\|u(\cdot, \tau)\|_{H^{2}}^{2}+\|\theta(\cdot, \tau)\|_{H^{2}}^{2}\right) d \tau\right) \\
\leq & C\left(T_{0}\right) \exp \left(C \int_{T_{0}}^{t}\|\nabla u(\cdot, \tau)\|_{\dot{B}_{\infty, \infty}^{-1}}^{2} d \tau \sup _{T_{0} \leq \tau \leq t} \log \left(\|u(\cdot, \tau)\|_{H^{2}}^{2}+\|\theta(\cdot, \tau)\|_{H^{2}}^{2}\right)\right) \\
\leq & C\left(T_{0}\right) \exp \left(C \int_{T_{0}}^{t}\|\nabla u(\cdot, \tau)\|_{\dot{B}_{\infty, \infty}^{-1}}^{2} d \tau \log \sup _{T_{0}<\tau \leq t}\left(\|u(\cdot, \tau)\|_{H^{2}}^{2}+\|\theta(\cdot, \tau)\|_{H^{2}}^{2}\right)\right) \\
\leq & C\left(T_{0}\right) \exp (C \varepsilon \log F(t)) \\
\leq & C\left(T_{0}\right)[F(t)]^{C \varepsilon}
\end{aligned}
$$

where

$$
C\left(T_{0}\right)=C\left(\left\|\nabla u\left(\cdot, T_{0}\right)\right\|_{L^{2}}^{2}+\left\|\nabla \theta\left(\cdot, T_{0}\right)\right\|_{L^{2}}^{2}\right) .
$$

Next, applying $\Delta$ to the equations $(1.1)_{1},(1.1)_{2}$, taking the $L^{2}$ inner product of the obtained equations with $-\Delta u$ and $-\Delta \theta$, respectively, adding them up and using 
the incompressible conditions $\nabla \cdot u=0$, we arrive at

$$
\begin{aligned}
& \frac{1}{2} \frac{d}{d t}\left(\|\Delta u\|_{L^{2}}^{2}+\|\Delta \theta\|_{L^{2}}^{2}\right)+\left\|\nabla^{3} u\right\|_{L^{2}}^{2}+\left\|\nabla^{3} \theta\right\|_{L^{2}}^{2} \\
= & \int_{\mathbb{R}^{3}} \Delta\left(\theta e_{3}\right) \cdot \Delta u d x-\int_{\mathbb{R}^{3}} \Delta(u \cdot \nabla u) \cdot \Delta u d x-\int_{\mathbb{R}^{3}} \Delta(u \cdot \nabla \theta) \cdot \Delta \theta d x \\
\leq & \left|\int_{\mathbb{R}^{3}} \Delta\left(\theta e_{3}\right) \cdot \Delta u d x\right|+\left|\int_{\mathbb{R}^{3}}(\Delta u \cdot \nabla u) \cdot \Delta u d x\right|+2 \sum_{i=1}^{3}\left|\int_{\mathbb{R}^{3}}\left(\partial_{i} u \cdot \nabla \partial_{i} u\right) \cdot \Delta u d x\right| \\
& +\left|\int_{\mathbb{R}^{3}}(\Delta u \cdot \nabla \theta) \cdot \Delta \theta d x\right|+2 \sum_{i=1}^{3}\left|\int_{\mathbb{R}^{3}}\left(\partial_{i} u \cdot \nabla \partial_{i} \theta\right) \cdot \Delta \theta d x\right| \\
= & \sum_{k=1}^{5} A_{k} .
\end{aligned}
$$

Now we will estimate the terms on the right-hand side of (3.15) one by one as follows. Let us begin with estimating the term $A_{1}$.

Using Lemma 2.4 with $p=q=r=j=2, k=3$ and $m=1, A_{1}$ can be bounded above as follows:

$$
\begin{aligned}
A_{1} & \leq C\|\Delta u\|_{L^{2}}\|\Delta \theta\|_{L^{2}} \\
& \leq C\|\nabla u\|_{L^{2}}^{\frac{1}{2}}\left\|\nabla^{3} u\right\|_{L^{2}}^{\frac{1}{2}}\|\nabla \theta\|_{L^{2}}^{\frac{1}{2}}\left\|\nabla^{3} \theta\right\|_{L^{2}}^{\frac{1}{2}} \\
& =\left(\left\|\nabla^{3} u\right\|_{L^{2}}^{2}\right)^{\frac{1}{4}}\left(\left\|\nabla^{3} \theta\right\|_{L^{2}}^{2}\right)^{\frac{1}{4}}\left(C\|\nabla u\|_{L^{2}}\|\nabla \theta\|_{L^{2}}\right)^{\frac{1}{2}} \\
& \leq \frac{1}{16}\left\|\nabla^{3} u\right\|_{L^{2}}^{2}+\frac{1}{16}\left\|\nabla^{3} \theta\right\|_{L^{2}}^{2}+C\|\nabla u\|_{L^{2}}\|\nabla \theta\|_{L^{2}} \\
& \leq \frac{1}{16}\left\|\nabla^{3} u\right\|_{L^{2}}^{2}+\frac{1}{16}\left\|\nabla^{3} \theta\right\|_{L^{2}}^{2}+C\left(\|\nabla u\|_{L^{2}}^{2}+\|\nabla \theta\|_{L^{2}}^{2}\right) .
\end{aligned}
$$

Let us now recall Gagliardo-Nirenberg's inequality

$$
\|\Delta f\|_{L^{4}} \leq C\|\nabla f\|_{L^{2}}^{\frac{1}{8}}\left\|\nabla^{3} f\right\|_{L^{2}}^{\frac{7}{8}}
$$

Thus, we obtain

$$
\begin{aligned}
A_{2}, A_{3} & \leq C\|\nabla u\|_{L^{2}}\|\Delta u\|_{L^{4}}^{2} \\
& \leq C\|\nabla u\|_{L^{2}}\|\nabla u\|_{L^{2}}^{\frac{1}{4}}\left\|\nabla^{3} u\right\|_{L^{2}}^{\frac{7}{4}} \\
& =C\|\nabla u\|_{L^{2}}^{\frac{5}{4}}\left\|\nabla^{3} u\right\|_{L^{2}}^{\frac{7}{4}}=\left(C\|\nabla u\|_{L^{2}}^{10}\right)^{\frac{1}{8}}\left(\left\|\nabla^{3} u\right\|_{L^{2}}^{2}\right)^{\frac{7}{8}} \\
& \leq \frac{1}{16}\left\|\nabla^{3} u\right\|_{L^{2}}^{2}+C\|\nabla u\|_{L^{2}}^{10} .
\end{aligned}
$$


Similarly to the estimate of $A_{1}$, the terms $A_{4}$ and $A_{5}$ can be bounded above as

$$
\begin{aligned}
A_{4}, A_{5} & \leq C\|\nabla \theta\|_{L^{2}}\|\Delta \theta\|_{L^{4}}\|\Delta u\|_{L^{4}} \\
& \leq C\|\nabla \theta\|_{L^{2}}\left(\|\Delta u\|_{L^{4}}^{2}+\|\Delta \theta\|_{L^{4}}^{2}\right) \\
& \leq C\|\nabla \theta\|_{L^{2}}\|\nabla u\|_{L^{2}}^{\frac{1}{4}}\left\|\nabla^{3} u\right\|_{L^{2}}^{\frac{7}{4}}+C\|\nabla \theta\|_{L^{2}}^{\frac{5}{4}}\left\|\nabla^{3} \theta\right\|_{L^{2}}^{\frac{7}{4}} \\
& \leq \frac{1}{4}\left\|\nabla^{3} u\right\|_{L^{2}}^{2}+C\|\nabla \theta\|_{L^{2}}^{8}\|\nabla u\|_{L^{2}}^{2}+\frac{1}{2}\left\|\nabla^{3} \theta\right\|_{L^{2}}^{2}+C\|\nabla \theta\|_{L^{2}}^{10} \\
& \leq \frac{1}{16}\left\|\nabla^{3} u\right\|_{L^{2}}^{2}+\frac{1}{4}\left\|\nabla^{3} \theta\right\|_{L^{2}}^{2}+C\|\nabla \theta\|_{L^{2}}^{8}\left(\|\nabla u\|_{L^{2}}^{2}+\|\nabla \theta\|_{L^{2}}^{2}\right) .
\end{aligned}
$$

Summarizing all the estimates and absorbing the dissipative term, we can derive

$$
\begin{aligned}
\frac{d}{d t}\left(\|\Delta u\|_{L^{2}}^{2}+\|\Delta \theta\|_{L^{2}}^{2}\right) & \leq C\|\nabla u\|_{L^{2}}^{10}+C\|\nabla \theta\|_{L^{2}}^{8}\left(\|\nabla u\|_{L^{2}}^{2}+\|\nabla \theta\|_{L^{2}}^{2}\right) \\
& \leq C\left(\|\nabla u\|_{L^{2}}^{8}+\|\nabla \theta\|_{L^{2}}^{8}\right)\left(\|\nabla u\|_{L^{2}}^{2}+\|\nabla \theta\|_{L^{2}}^{2}\right) \\
& \leq C\left(\|\nabla u\|_{L^{2}}^{2}+\|\nabla \theta\|_{L^{2}}^{2}\right)^{4}\left(\|\nabla u\|_{L^{2}}^{2}+\|\nabla \theta\|_{L^{2}}^{2}\right) \\
& \leq C\left(\|\nabla u\|_{L^{2}}^{2}+\|\nabla \theta\|_{L^{2}}^{2}\right)^{5} \\
& \leq C\left(T_{0}\right)[F(t)]^{5 C \varepsilon} .
\end{aligned}
$$

Integrating the above estimate over interval $\left(T_{0}, t\right)$ and observing that $F(t)$ is a monotonically increasing function, we thus have

$$
\begin{aligned}
& \|\Delta u(\cdot, t)\|_{L^{2}}^{2}+\|\Delta \theta(\cdot, t)\|_{L^{2}}^{2} \\
\leq & \left\|\Delta u\left(\cdot, T_{0}\right)\right\|_{L^{2}}^{2}+\left\|\Delta \theta\left(\cdot, T_{0}\right)\right\|_{L^{2}}^{2}+C\left(T_{0}\right) \int_{T_{0}}^{t}[F(\tau)]^{5 C \varepsilon} d \tau .
\end{aligned}
$$

By using (3.14), it follows that

$$
\begin{aligned}
F(t) & \leq\left\|u\left(\cdot, T_{0}\right)\right\|_{H^{2}}^{2}+\left\|\theta\left(\cdot, T_{0}\right)\right\|_{H^{2}}^{2}+C \int_{T_{0}}^{t}[F(\tau)]^{5 C \varepsilon} d \tau \\
& \leq\left\|u\left(\cdot, T_{0}\right)\right\|_{H^{2}}^{2}+\left\|\theta\left(\cdot, T_{0}\right)\right\|_{H^{2}}^{2}+C\left(T_{0}\right)\left(t-T_{0}\right)[F(t)]^{5 C \varepsilon} .
\end{aligned}
$$

Choosing $\varepsilon$ such that $5 C \varepsilon<1$, the above inequality yields for any $t \in\left[T_{0}, T\right)$

$$
F(t) \leq C<\infty,
$$

which implies that $(u, \theta) \in L^{\infty}\left(0, T ; H^{1}\left(\mathbb{R}^{3}\right)\right) \cap L^{2}\left(0, T ; H^{2}\left(\mathbb{R}^{3}\right)\right)$. This completes the proof of Theorem 1.1.

Remark 3.1 Comparing our result with [27], we have simplified the proof of Theorem 1.1 in [27], in fact we only need $\mathrm{H}^{2}$ a priori estimates of solutions. 


\section{References}

1. H. Abidi and T. Hmidi. On the global well-posedness for Boussinesq system. J. Differential Equations, 233(1):199-220, 2007.

2. Jean-Michel Bony. Calcul symbolique et propagation des singularités pour les équations aux dérivées partielles non linéaires. Ann. Sci. École Norm. Sup. (4), 14(2):209-246, 1981.

3. J. R. Cannon and Emmanuele DiBenedetto. The initial value problem for the Boussinesq equations with data in $L^{p}$. In Approximation methods for Navier-Stokes problems (Proc. Sympos., Univ. Paderborn, Paderborn, 1979), volume 771 of Lecture Notes in Math., pages 129-144. Springer, Berlin, 1980.

4. Dongho Chae, Sung-Ki Kim, and Hee-Seok Nam. Local existence and blow-up criterion of Hölder continuous solutions of the Boussinesq equations. Nagoya Math. J., 155:55-80, 1999.

5. Dongho Chae and Hee-Seok Nam. Local existence and blow-up criterion for the Boussinesq equations. Proc. Roy. Soc. Edinburgh Sect. A, 127(5):935-946, 1997.

6. Jean-Yves Chemin. Perfect incompressible fluids, volume 14 of Oxford Lecture Series in Mathematics and its Applications. The Clarendon Press, Oxford University Press, New York, 1998. Translated from the 1995 French original by Isabelle Gallagher and Dragos Iftimie.

7. Raphaël Danchin and Marius Paicu. Existence and uniqueness results for the Boussinesq system with data in Lorentz spaces. Phys. D, 237(10-12):1444-1460, 2008.

8. Raphaël Danchin and Marius Paicu. Les théorèmes de Leray et de Fujita-Kato pour le système de Boussinesq partiellement visqueux. Bull. Soc. Math. France, 136(2):261-309, 2008.

9. Miguel Escobedo and Enrike Zuazua. Large time behavior for convection-diffusion equations in $\mathbf{R}^{N}$. J. Funct. Anal., 100(1):119-161, 1991.

10. Jishan Fan and Tohru Ozawa. Regularity criteria for the 3D density-dependent Boussinesq equations. Nonlinearity, 22(3):553-568, 2009.

11. Jishan Fan and Yong Zhou. A note on regularity criterion for the 3D Boussinesq system with partial viscosity. Appl. Math. Lett., 22(5):802-805, 2009.

12. Sadek Gala, Zhengguang Guo, and Maria Alessandra Ragusa. A remark on the regularity criterion of Boussinesq equations with zero heat conductivity. Appl. Math. Lett., 27:70-73, 2014.

13. Sadek Gala and Maria Alessandra Ragusa. Logarithmically improved regularity criterion for the Boussinesq equations in Besov spaces with negative indices. Appl. Anal., 95(6):1271-1279, 2016.

14. Zhengguang Guo and Sadek Gala. Regularity criterion of the Newton-Boussinesq equations in $\mathbb{R}^{3}$. Commun. Pure Appl. Anal., 11(2):443-451, 2012.

15. Hideo Kozono, Takayoshi Ogawa, and Yasushi Taniuchi. The critical Sobolev inequalities in Besov spaces and regularity criterion to some semi-linear evolution equations. Math. Z., 242(2):251-278, 2002.

16. Shuji Machihara and Tohru Ozawa. Interpolation inequalities in Besov spaces. Proc. Amer. Math. Soc., 131(5):1553-1556, 2003.

17. Andrew Majda. Introduction to PDEs and waves for the atmosphere and ocean, volume 9 of Courant Lecture Notes in Mathematics. New York University, Courant Institute of Mathematical Sciences, New York; American Mathematical Society, Providence, RI, 2003.

18. Yves Meyer. Oscillating patterns in some nonlinear evolution equations. In Mathematical foundation of turbulent viscous flows, volume 1871 of Lecture Notes in Math., pages 101-187. Springer, Berlin, 2006.

19. Joseph Pedlosky. Geophysical Fluids Dynamics. Springer Verlag, New York, 1979.

20. Yuming Qin, Xinguang Yang, Yu-Zhu Wang, and Xin Liu. Blow-up criteria of smooth solutions to the 3D Boussinesq equations. Math. Methods Appl. Sci., 35(3):278-285, 2012.

21. Hua Qiu, Yi Du, and Zheng'an Yao. A blow-up criterion for 3D Boussinesq equations in Besov spaces. Nonlinear Anal., 73(3):806-815, 2010.

22. Elias M. Stein. Singular integrals and differentiability properties of functions. Princeton Mathematical Series, No. 30. Princeton University Press, Princeton, N.J., 1970.

23. Hans Triebel. Theory of function spaces, volume 78 of Monographs in Mathematics. Birkhäuser Verlag, Basel, 1983.

24. Zhaoyin Xiang. The regularity criterion of the weak solution to the $3 \mathrm{D}$ viscous Boussinesq equations in Besov spaces. Math. Methods Appl. Sci., 34(3):360-372, 2011.

25. Fuyi Xu, Qian Zhang, and Xiaoxin Zheng. Regularity criteria of the 3D Boussinesq equations in the Morrey-Campanato space. Acta Appl. Math., 121:231-240, 2012. 
26. Zhuan Ye. Blow-up criterion of smooth solutions for the Boussinesq equations. Nonlinear Anal., 110:97-103, 2014

27. Zhuan Ye. A logarithmically improved regularity criterion of smooth solutions for the 3D Boussinesq equations. Osaka J. Math., 53(2):417-423, 2016. 